\title{
Warning of Potential Collision for Vehicles
}

\author{
Huang Yue*, Qin Gui He, Liu Tong, Sun Ning, Wang Xiao Dan \\ College of Computer Science and Technology/Center for Computer Fundamental Education, Jilin University, \\ Changchun, China
}

\begin{abstract}
A moving vehicle may very likely run into accidents. The occurrence rate of accidents would be largely reduced if the driver is warned in advance, even only $0.5 \mathrm{~s}$ earlier. For a running vehicle, the driving route within short time before collision has the characteristic of Markov. In this case, the coordinates of position only have to be considered within a short range, rather than the running status during the past long period. Within short period before collision, the driving route can be basically divided into two states: a straight line and a binomial curve. In this paper, a mechanism is proposed for sending collision warning messages to running vehicles.
\end{abstract}

Keywords: Curve fitting, collision, dissemination, emergency warning, VANET.

\section{INTRODUCTION}

Vehicular Ad-hoc NETwork (VANET) is an open mobile Ad-hoc network composed of intercommunications between vehicles and fixed access point on roads. VANET is a selforganized between-vehicle communication network with open structure [1].

VANET is widely used in road traffic safety, information service, traffic query, and assistant driving. In particular, research on road traffic safety focuses on anti-collision warning. The research on various traffic accidents conducted by German Benz Automobile Corporation shows that at least $60 \%$ of rear-end accidents would be prevented if the drivers could promptly recognize the risks and take measures only $0.5 \mathrm{~s}$ earlier. About $90 \%$ of traffic accidents can be avoided if measures are taken $1 \mathrm{~s}$ earlier. The National Highway Traffic Safety Administration sponsors developed an Integrated Vehicle-Based Safety System to monitor traffic environment with an aim to reducing crashes. A similar project sponsored by the European Union develops a precrash sensorial system [2-5].

Sengupta et al. [6] first proposed a cooperative driver assistance system, which considers varying emergency warning grades. This system has been applied in many aspects, such as intersection collision emergency warning or forward collision warning, with parameters such as collision time as the condition for sending Emergency warning messages (EWMs). The positioning error can be reduced below $50 \mathrm{~cm}$ by integrating GPS, yaw rate gyro, and steering angle sensor.

Tang et al. [7] investigated timings for collision avoidance systems assuming DSRC transmission delays of $25 \mathrm{~ms}$ and $300 \mathrm{~ms}$ in normal and poorer channel conditions, respectively. They concentrated on the events such as DSRC communication latency, detection range, road condition,

\footnotetext{
*Address correspondence to this author at the College of Computer Science and Technology/Center for Computer Fundamental Education, Jilin University, Changchun, China; Tel: +86-0431-85095375; E-mails: huangyue@jlu.edu.cn, qingh@jlu.edu.cn
}

driver reaction and deceleration rate. With these events, they defined two collision avoidance timings: critical time to avoid collision and preferred time to avoid collision.

The work reported in Mitropoulos et al. [8] also presents a driver assistant system (WILLWARN- wireless local danger warning) designed to timely warn the driver about a dangerous situation ahead by decentralized distribution of warnings and incident messages via ad hoc inter-vehicle communications.

The study reported in Ibanez-Guzman et al. [9] presents the implementation and basic test in an intersection scenario of an instance of the architecture developed for cooperative vehicle applications. The study highlighted the relevant impact of obstructions on the communications performance, the importance of positioning technologies, the difficulty in creating hazardous situations in testing scenarios, and the need to adequately quantify and log results.

An approach to the study of the positioning impact is that reported by Santa et al. [10]. The authors show that the most appropriate navigation sub-system is based on a combination of motion and GNS sensors. The studies evaluated the positioning error and proposed advanced positioning solutions to support cooperative V2X active safety applications, without addressing the design and implementation of complete functional driver assistant system prototypes.

Lefèvre et al. [11] pointed out that risk assessment at intersections is possible by comparing intention and expectation. Liebner et al. [12] also used drivers' intent inference at intersections.

In addition, from the aspect of data distribution, $\mathrm{T}$. Nadeem et al. [13] discussed dissemination techniques for the exchange of messages containing either traffic conditions or vehicles' current status information (e.g. speed and direction).

Dornbush et al. [14] focused on the application of informing vehicles about traffic conditions in their surroundings. They proposed a mechanism for vehicles to 
estimate traffic conditions and, through a clustering approach, disseminated the obtained information to vehicles in the network.

In this paper, a EWM transmission mechanism is built. The driving route has the characteristics of Markov: when risks occur, it is independent of the history of driving. Thus, to send emergency warning, it only depends on the driving state within short time. VANET can be used to obtain the position information of vehicles, thereby predicting the driving route, estimating the point of two-vehicle collision, and obtaining the time difference $[15,16]$. In this way, the vehicles in neighbouring queues are sorted out by emergency condition, and involve those reaching the alarm threshold in the queue to receive EWMs. Only vehicles entering this queue are regarded as the network nodes of EWM in VANET, which largely reduces load compared with the flooding type network.

\section{MODELING MECHANISM}

\subsection{Fitting of Driving Route}

The driving route of a vehicle can be divided into subsections depending on the condition of roads. Within a short time, the driving route may be classified into two types: straight line or polynomial curve.

1) The straight route can be expressed as $y=a x+b$. During the travelling, a vehicle periodically sends messages to other vehicles and promptly updates the position information in the neighbour list. The system continually corrects and fits the route accordingly. Because of the errors contained in the data, the least square method is used to fit the straight line. According to the measured historical data, $\left(x_{i}, y_{i}\right)(i=0,2 \ldots n)$, a running vehicle follows the Markov rule that the possibility of braking-caused collision is independent of the previous driving route, and thus the value of $n$ should be within the effective time limit, expressed as n'. Least square method is used to build the following equation:

$$
\sum_{i=0}^{n^{\prime}} \varepsilon_{i}^{2}=\sum_{i=0}^{n^{\prime}}\left(a x_{i}+b-y_{i}\right)^{2}
$$

This is a function of a and b, namely:

$$
f(a, b)=\sum_{i=0}^{n^{\prime}}\left(a x_{i}+b-y_{i}\right)^{2}
$$

This problem is now equal to finding the minimum of $\mathrm{f}(\mathrm{a}$, b) under the following condition:

$$
\begin{aligned}
& \left\{\begin{array}{l}
\frac{\partial f(a, b)}{\partial b}=0 \\
\frac{\partial f(a, b)}{\partial a}=0
\end{array}\right. \\
& \text { Then } \\
& \left\{\begin{array}{l}
2 \sum_{i=0}^{n^{\prime}}\left(a x_{i}+b-y_{i}\right)=0 \\
2 \sum_{i=0}^{n^{\prime}}\left(a x_{i}+b-y_{i}\right) \cdot x_{i}=0
\end{array}\right.
\end{aligned}
$$

After unfolding and reorganization, then

$$
\left\{\begin{array}{l}
a \sum_{i=0}^{n^{\prime}} x_{i}+b \cdot n^{\prime}=\sum_{i=0}^{n^{\prime}} y_{i} \\
a \sum_{i=0}^{n^{\prime}} x_{i}^{2}+b \sum_{i=0}^{n^{\prime}} x_{i}=\sum_{i=0}^{n^{\prime}} x_{i} y_{i}
\end{array}\right.
$$

The matrix form is:

$$
\left[\begin{array}{cc}
\sum_{i=0}^{n^{\prime}} x_{i} & n^{\prime} \\
\sum_{i=0}^{n^{\prime}} x_{i}^{2} & \sum_{i=0}^{n^{\prime}} x_{i}
\end{array}\right]\left[\begin{array}{l}
a \\
b
\end{array}\right]=\left[\begin{array}{c}
\sum_{i=0}^{n^{\prime}} y_{i} \\
\sum_{i=0}^{n^{\prime}} x_{i} y_{i}
\end{array}\right]
$$

Then the values of $a$ and $b$ are found of this equation set. When a new point $\left(\mathrm{x}_{\text {new }}, \mathrm{y}_{\text {new }}\right)$ is obtained, $\mathrm{xnew}$ is substituted; if $\mathrm{y}=\mathrm{y}_{\text {new }}$ or $\mathrm{y}=\mathrm{y}_{\text {new }}+\varepsilon$ ( $\varepsilon$ is the permissible error), the driving route can be judged as a straight line. Otherwise, the route is a curve.

2) The polynomial curve can be expressed as $y=\sum_{k=0}^{m} a_{k} X^{k}:$ This is function of driving route.

From the historical data, the vehicle's coordinates can be obtained as set $\mathrm{A}=\left\{\left(\mathrm{x}_{0}, \mathrm{y}_{0}\right),\left(\mathrm{x}_{1}, \mathrm{y}_{1}\right) \ldots . .\left(\mathrm{x}_{\mathrm{m}}, \mathrm{y}_{\mathrm{m}}\right)\right\}$. The value of $\mathrm{m}$ should be within the effective time limit, expressed as $\mathrm{m}^{\prime}$. Then solving the fitting curve of $\mathrm{y}$ as equal to minimizing the sum of squared deviations of $y$ and $y_{m}$, namely:

$\Phi(X)=\sum_{i=0}^{m^{\prime}}\left[\sum_{k=0}^{n} a_{k} x_{i}^{k}-y_{i}\right]^{2}=\operatorname{Min}$

Then solving $\Phi(X)$ is transformed to finding the extremums, then:

$\frac{\partial \Phi(X)}{\partial a_{j}}=2 \sum_{i=0}^{m^{\prime}}\left(\sum_{k=0}^{n} a_{k} x_{i}^{k}-y_{i}\right) x_{i}^{j}=0 \quad \mathrm{j}=0,1,2,3 \ldots \mathrm{n}$

Unfolding Eq. (8), then

$\sum_{i=0}^{m^{\prime}} \sum_{k=0}^{n} a_{k} x_{i}^{k}=\sum_{k=0}^{n}\left(\sum_{i=0}^{m^{\prime}} x_{i}^{k+j}\right) a_{k}=\sum_{i=0}^{m^{\prime}} y_{i} x_{i}^{j}$

The above equation is equivalent to a linear equation set related to $\mathrm{a}_{0}-\mathrm{a}_{\mathrm{n}}$ :

$$
\left[\begin{array}{cccc}
m^{\prime} & \sum_{i=0}^{m^{\prime}} x_{i} & \ldots & \sum_{i=0}^{m^{\prime}} x_{i}^{n} \\
\sum_{i=0}^{m^{\prime}} x_{i} & \sum_{i=0}^{m^{\prime}} x_{i}^{2} & \ldots & \sum_{i=0}^{m^{\prime}} x_{i}^{n+1} \\
\vdots & \vdots & \vdots & \vdots \\
\sum_{i=0}^{m^{\prime}} x_{i}^{n} & \sum_{i=0}^{m^{\prime}} x_{i}^{n+1} & \ldots & \sum_{i=0}^{m^{\prime}} x_{i}^{2 n}
\end{array}\right]\left[\begin{array}{c}
a_{0} \\
a_{1} \\
\vdots \\
a_{n}
\end{array}\right]=\left[\begin{array}{c}
\sum_{i=0}^{m^{\prime}} y_{i} \\
\sum_{i=0}^{m^{\prime}} x_{i} y_{i} \\
\vdots \\
\sum_{i=0}^{m^{\prime}} x_{i}^{n} y_{i}
\end{array}\right]
$$

According to the properties of symmetric positive definite matrix, there is one and only solution to $\mathrm{a}_{0}-\mathrm{a}_{\mathrm{n}}$, which can determine the fitted nonlinear polynomial equation of driving vehicles. 


\subsection{EWM Sending Mechanism}

Based on the above route analysis, the collision point can be located. The route of collision may be a combination of straight line and curve. According to the travelling characteristics, beforehand, when collision occurs, if the curve route is adopted within short time, the route can be fitted using a quadratic polynomial curve, as shown in the Fig. (1) below:

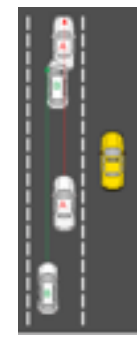

(a) Straight

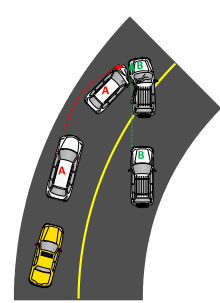

(b) Curve
Fig. (1). Driving conditions.

Then the two-vehicle intersection is equal to combinatorial solving $\mathrm{y}=\mathrm{ax}+\mathrm{b}$ and $\mathrm{y}=\mathrm{a}_{0}+\mathrm{a}_{1} \mathrm{x}+\mathrm{a}_{2} \mathrm{x}^{2}$, while their parameters are known before fitting.

If the equation set has no solution, the two vehicles will not collide; if it has two solutions, let $\left(\mathrm{x}_{1}, \mathrm{y}_{1}\right),\left(\mathrm{x}_{2}, \mathrm{y}_{2}\right)$; if it has one solution, then $\mathrm{x}_{1}=\mathrm{x}_{2}, \mathrm{y}_{1}=\mathrm{y}_{2}$. The nearest point of intersection ahead of the driving direction is a potential collision point, expressed as $\left(\mathrm{x}_{\text {cross }}, \mathrm{y}_{\text {cross }}\right)$. If there is no point of intersection, the two vehicles will not collide.

With the intersection between a straight line and a curve as an example, vehicle $\mathrm{A}$ is supposed to be located at $\left(\mathrm{x}_{\mathrm{A}}\right.$, $\mathrm{y}_{\mathrm{A}}$ ), running at a speed of $\mathrm{v}_{\mathrm{A}}$ along a straight line. Vehicle $\mathrm{B}$ located at $\left(\mathrm{x}_{\mathrm{B}}, \mathrm{y}_{\mathrm{B}}\right)$, running at a speed of $\mathrm{v}_{\mathrm{B}}$ along a curve. A and $\mathrm{B}$ are at distance of $\mathrm{s}_{\mathrm{A}}$ and $\mathrm{s}_{\mathrm{B}}$ respectively away from the cross point. Then

$s_{A}=\left|x_{\text {cross }}-x_{A}\right| /|\cos (\arctan (\alpha))|$

$s_{B}=\int_{x_{B}}^{x_{c r o s s}} \sqrt{1+\left(a_{1}+2 a_{2} x\right)^{2} d x}$

The time difference when $\mathrm{A}$ and $\mathrm{B}$ arrive at the cross point is $t_{\Delta}=\left|s_{A} / v_{A}-s_{B} / v_{B}\right| \leq \zeta$, where $\zeta$ is the warning threshold, and then EWM is sent.

\section{MESSAGE SENDING MECHANISM}

When two vehicles intercommunicate, the originating node $\mathrm{S}_{0}$ periodically sends a message package "Hello" to the neighbor nodes within its transmission range. The message "Hello" contains the information about the position of this sender node and relevant information collected from the newest neighbor nodes. By exchanging the message "Hello", each node can build a newest information list for its neighbor nodes, and understand the distribution of neighbor nodes within its transmission range. Then $\mathrm{S}_{0}$ can maintain the network topology with these neighbor nodes. Supposing A-H are the neighbor vehicles within a two-hop range of $\mathrm{S}_{0}$. If a message is sent out in the flooding way, there will be redundant information in the network. Therefore, to reduce the redundant EWM, the following method is proposed to send
EWM. When $\mathrm{S}_{0}$ to send a warning of braking, at the first hop, $\mathrm{S}_{0}$ selects the nodes with which the collision possibility is within a certain threshold; however, these nodes may become the nodes at the second hop. Transmission is not necessary if no node is within the threshold. After the above computation, $\mathrm{A}, \mathrm{B}, \mathrm{C}, \mathrm{D}, \mathrm{E}$ and $\mathrm{F}$ are supposed to be neighbor nodes of $\mathrm{S}_{0}$, and $\mathrm{G}, \mathrm{H}$ as neighbor nodes of $\mathrm{B}$. In the flooding mode, these nodes will retransmit the message extensively (as shown in Fig. 2). Then the above threshold computation is introduced to avoid such circumstance. If among all neighbor nodes of $\mathrm{S}_{0}$, only $\mathrm{B}$ is within the threshold, then B becomes a retransmitting node. B will compute its transmission queue with the same method. If $\mathrm{G}$ is within the threshold, $\mathrm{G}$ becomes the second-hop node. Transmission will be stopped if no node in G's transmission queue is within the threshold (as shown in Fig. 3).

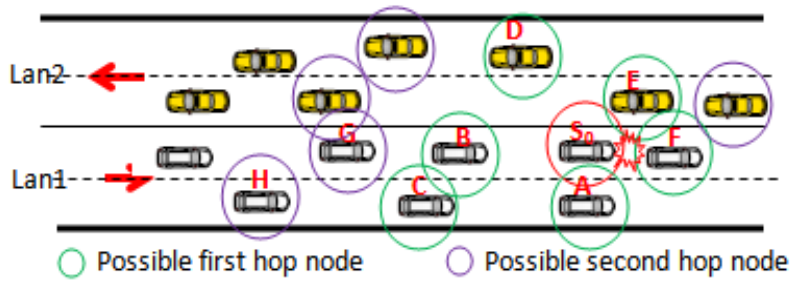

Fig. (2). Flooding mode.

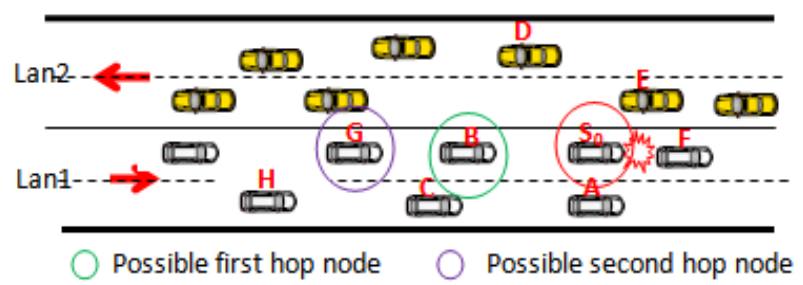

Fig. (3). EWM mode.

Obviously, the amount of transmitted data will be reduced largely. The nodes within the emergency warning range can be calculated as Algorithm 1.

The steps are as follows: based on the function fitting method in Section 1.2, the records of travelling are transferred to generate a neighbor queue travelling route including the originating node and fit a function. The transmitting queue is selected with the between-vehicle distance as threshold, and involves the vehicles within the warning vehicle-distance into the computation. First, the fitting function is computed for the neighbor vehicles with the smallest space in between, and the collision time is estimated; if it is within the threshold, then EWM is sent, and moving on to the next neighbor node, the above computation is repeated. The originating node searches its transmission queue L, sends the EWM to the neighbor node S' within the time threshold; when S' receives the EWM, it searches its own sending queue $\mathrm{L}$; if there is no node in $\mathrm{L}$, no node is within the warning range, then sending of EWM is stopped to the next node.

\section{SIMULATION}

The Vanet simulation system is composed of two modules: network communication module and vehicle motion module. 
Algorithm 1. Algorithm for the choice of the warning range nodes.

Warning_options_algorithm( )

1 Traverse_Neighbor $\left(\mathrm{s}_{0}\right) / /$ Search the whole neighbor queue of node $\mathrm{s}$

2 If $\mathrm{d} \leq \varepsilon$

$3 \mathrm{~L} \leftarrow \mathrm{s} ; / /$ select the neighbor nodes with between-vehicle distance within the threshold, and form a queue L; Sort in the ascending order

4 while S' $\leftarrow \mathrm{L}$ do $/ /$ Find the first node of queue $\mathrm{L}$, obtain n-step historical position information $\left(\mathrm{x}_{1}, \mathrm{y}_{1}\right),\left(\mathrm{x}_{2}, \mathrm{y}_{2}\right), \ldots$

$5 \mathrm{dl} \leftarrow(\mathrm{x} 1, \mathrm{y} 1)$;

$6 \mathrm{~d} 2 \longleftarrow\left(\mathrm{x}_{2}, \mathrm{y}_{2}\right) ; \ldots$

7 Function fitting $\left(\mathrm{d}_{1}, \mathrm{~d}_{2}, \ldots\right)$;// Transfer the fitting algorithm to produce a function set

8 Time_calculation(s');// Compute the time difference

9 if $t \Delta\left(\mathrm{s}^{\prime}, \mathrm{s}_{0}\right) \leq / /$ The time difference between the originating node $\mathrm{s}_{0}$ ' and other vehicles is within the threshold.

10 send(message);

11 delete(s');

12 next;

13 end while;

\subsection{Simulation Environment of Network and Road Traffic}

The network simulation module simulates the communication between vehicle nodes. In this paper, this module is realized via OMNET++. At present, VANET vehicle-targeted electronic wireless communication accords to $802.11 \mathrm{p}$ communication agreement. Veins possess complete and detailed IEEE 802.11p and IEEE 1609.4 DSRC / WAVE network layer models and creditable vehicle fluidity model. Therefore, the whole simulation model is based on OMNet++, and with the introduction of Veins frame, the road traffic is simulation displayed on SUMO.

\subsection{Performance Assessment}

The Jilin University campus map, double-channel road is introduced which induces probability of random collision. Vehicle speed changes randomly at 20- $40 \mathrm{~m} / \mathrm{h}$. Two types of messages including periodical message and EWM are sent. The periodical messages are generated at $10 \mathrm{pkts} / \mathrm{s}$; EWM is generated when the traveling speed reaches the above threshold. The traveling simulation scene is as follows:

The simulation experiments accord to EWM sent by DCF competition mechanism in IEEE 802.11MAC agreement. The simulation time is $600 \mathrm{~s}$ and the size of EWM is 1024 Byte (Fig. 4).

The average delay of EWM transmission increases along with the elevated vehicle density, and the amplitude is more obvious (as shown in Fig. 5). When the number of vehicles is smaller than 20, the average delay of EWM transmission is intensified very smoothly, but when the number is larger than 20, the average delay is intensified at a higher amplitude.

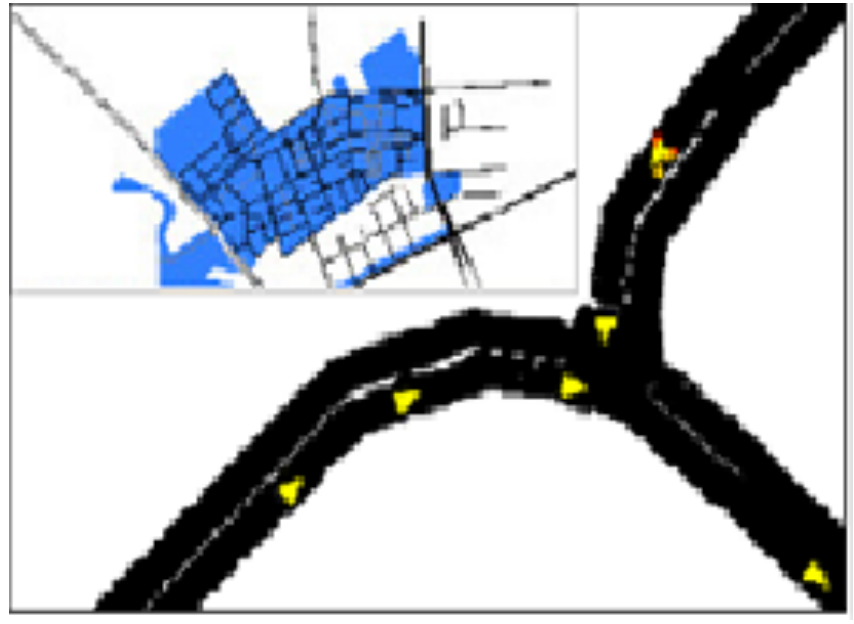

Fig. (4). Traveling simulation scene.

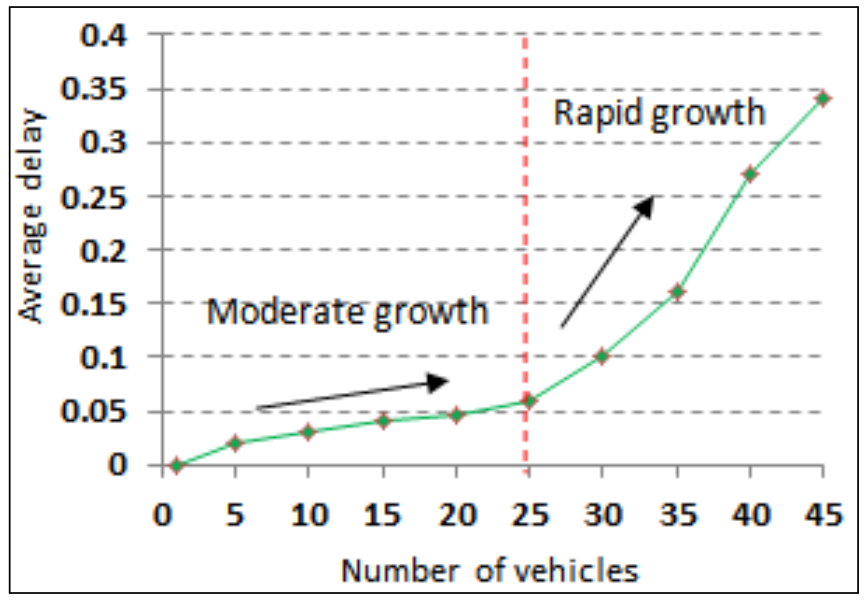

Fig. (5). The relationship of the traffic density and the average delay(s). 


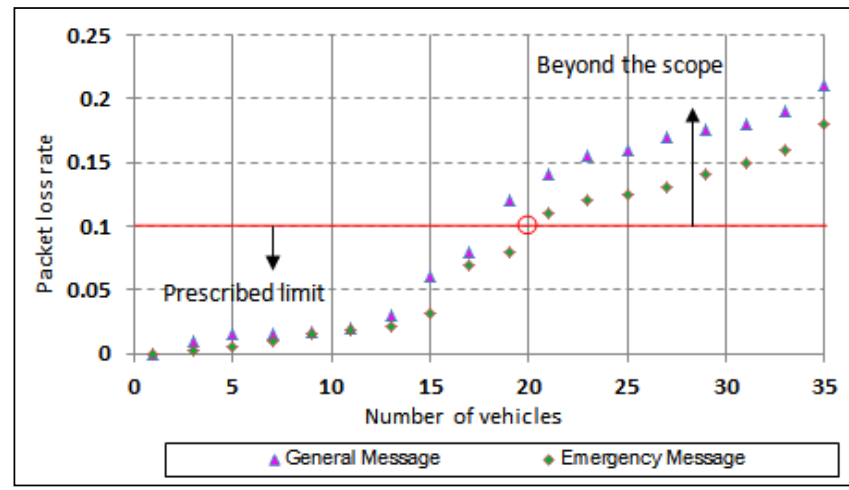

Fig. (6). The drop rate variation with the number of vehicles.

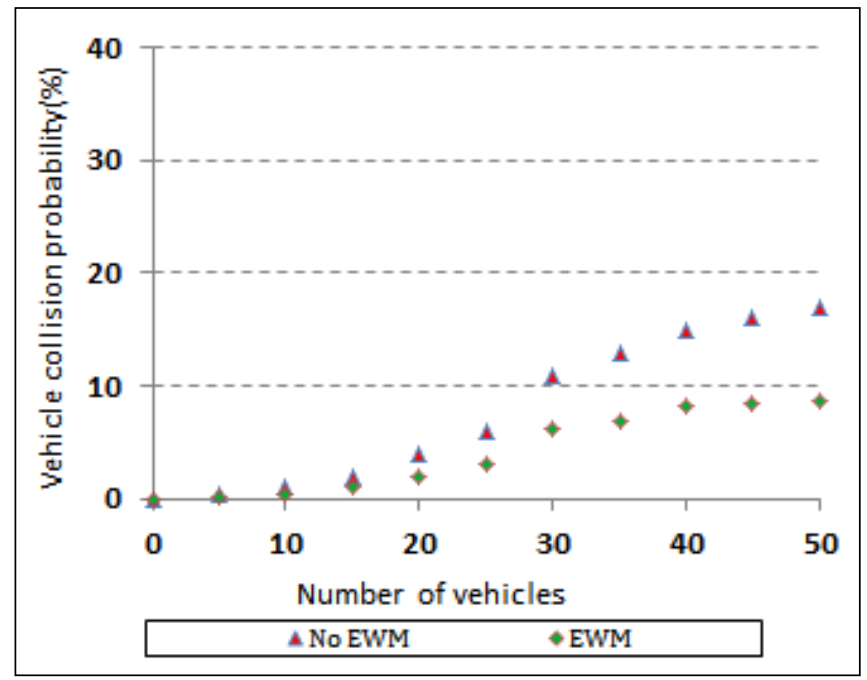

Fig. (7). Vehicle collision probability comparison.

Fig. (6) shows the package drop rate along with the number of vehicles. The drop rate increases with the increased number of vehicles; when there is only 1 vehicle in the communication extent, the drop rate of emergency message is 0 , the drop rate of common message is $0.5 \%$ which is due to internal collision of messages. Along with the increased number of vehicles in the communication range, the message collision is intensified, leading to a greater number of message retransmission and higher package drop rate. The demand of package drop rate does not exceed $10 \%$, and the maximum vehicle number within the emergency message communication time limit is no greater than 20 .

Fig. (7) compares the variations of vehicle number along with the vehicle collision probability under two situations: EWM and No EWM. Generally, the vehicle collision probability becomes higher with the increased number of vehicles under both situations, but the amplitude becomes gradually smaller. When the number of vehicles reaches 30 , the probability of vehicle collision gradually stabilizes.

\section{CONCLUSION AND PROSPECTS}

First the background and meaning of research on development and technology of VANET warning data are introduced and a warning model mechanism is built. Targeted at the distributing speed and credibility of warning data, a warning data distributing mechanism suitable for VANET is proposed. This mechanism mainly considers single-hop transmission of warning data and its role in emergency warning to avoid traffic accidents.

Many meaningful findings are achieved but further improvement and perfection are needed.

1) The newly built system is limited and restricted to simplify the simulation scene. Actual measurements will be conducted to validate the effects of this mechanism.

2) It is very important to apply VANET in other aspects, such as assisting vehicles to understand position information of relevant service network points, improving the comfort of drivers, and providing services of mobile office and on-line entertainment. These aspects will be studied in the future.

\section{CONFLICT OF INTEREST}

The authors confirm that this article content has no conflict of interest.

\section{ACKNOWLEDGEMENTS}

In this paper, the research was sponsored by the State Ministry of industry and Information Technology development of IOT special in 2011:Vehicle information system to support the car network and application demonstration; Key scientific and technological projects of Jilin Province in 2014: Research on Fasting Detecting method of Beef Quality Based on Bionic oral sensory system.

\section{REFERENCES}

[1] A.Sulaiman, S.V. K. Raja, and S. H. Park, "Improving scalability in vehicular communication using one-way hash chain method," Ad Hoc Networks, 2013

[2] B. R. Devi, K. K.Rao, and M. A. Rani, "Avoidance of vehicular collision and implementation of automatic path diversion in emergency for multi-hop vanets using cooperative relay," Wireless Communication, vol. 7, no. 2, pp.44-50, 2015.

[3] M. Hadded, R. Zagrouba, A.Laouiti, P.Muhlethaler, and L.A. Saidane, "An optimal strategy for collision-free slots allocations in vehicular ad-hoc networks,"Advances in Intelligent Systems and Computing, vol. 306, pp.15-30, 2015.

[4] M. Ito, "Development of INter-vehicle communication type driving support system in the ASV-3 project," Mitsubishi Motors Technical Review, 2007.

[5] K. Fuerstenberg, P. Baraund, G.Caporaletti, S. Citelli, Z. Eitan, U.Lages, and C. Lavergne, "Development of a pre-crash sensorial system," The CHAMELEON Project, p. 23, 2002.

[6] R..Sengupta, S. Rezaei, S.E. Shladover, D.Cody, S.Dickey, and H.Krishnan, "Cooperative collision warning systems: concept definition and experimental implementation," Journal of Intelligent Transportation Systems, vol. 11, no. 3, pp.143-155, 2007.

[7] A. Tang, and A. Yip, "Collision avoidance timing analysis of dsrcbased vehicles. accident analysis and prevention," vol. 42, no. 1, pp.182-195, 2010.

[8] G.K. Mitropoulos, I.S.Karanasiou, A.Hinsberger, F. AguadoAgelet, H.Wieker, H..J. Hilt, S. Mammar, and G.Noecker, "Wireless local danger warning: cooperative foresighted driving using intervehicle communication," IEEE Transactions on Intelligent Transportation System, vol. 11, no. 3, pp. 539- 553,

[9] Guzman, J. Lefevre, S. Mokkadem, and A. S. Rodhaim, "Vehicle to vehicle communications applied to road intersection safety, field 
results," Conference on Intelligent Transportation Systems (ITSC), 2010, pp. 192-197.

[10] J. Santa, R..Toledo-Moreo, M.A. Zamora-Izquierdo, B. Úbeda, and A.F. Gómez-Skarmeta, "An analysis of communication and navigation issues in collision avoidance support systems," Elsevier Transportation Research Part C - Emerging Technologies, vol.18, no. 3, pp.351-366,2011.

[11] S. Lefevre, C. Laugier, and J. Ibanez-Guzman, "Risk assessment at road intersections: comparing intention and expectation", IEEE Intelligent Vehicles Symposium, 2012, pp. 165-171.

[12] M. Liebner, M. Baumann, F. Klanner, and C. Stiller, "Driver Intent Inference at Urban Intersections using the Intelligent Driver Model”, In: IEEE Intelligent Vehicles Symposium (IV 2012), 2012, pp. 1162-167.

[13] T. Nadeem, P. Shankar, and L. Iftode, "A comparative study of data dissemination models for vanets," In: Annual International
Conference on Mobile and Ubiquitous Systems-Workshops, 2006, pp. 1-10.

[14] S. Dornbush, A. Joshi, "Streetsmart traffic: discovering and disseminating automobile congestion using vanet's", Vehicular Technology Conference, 2007, pp. 11-15.

[15] S.Feng, J. W. Cheong, G. Andrew, "An ultra-wide bandwidthbased range/GPS tight integration approach for relative positioning in vehicular ad hoc networks,"Measurement Science and Technology, vol. 26, no. 4, pp. 45003-45014, 2015.

[16] N. Akhtar, and O. Ozkasap, "Vehicle mobility and communication channel models for realistic and efficient highway VANET simulation," Vehicular Technology, vol. 64, no. 1, pp. 248-262, 2012.

(C) Yue et al.; Licensee Bentham Open

This is an open access article licensed under the terms of the (https://creativecommons.org/licenses/by/4.0/legalcode), which permits unrestricted, non-commercial use, distribution and reproduction in any medium, provided the work is properly cited. 